\title{
Semi-Prone Thoracoscopic Esophagectomy for Esophageal Carcinoma with Aberrant Right Subclavian Artery and Non-Recurrent Inferior Laryngeal Nerve: A Case Report
}

Kazunori Koyama ( $\nabla$ kznr.kym@gmail.com )

Yokohama Sakae Kyosai Hospital: Yokohama Sakae Kyosai Byoin

Toru Watanabe

Yokohama Sakae Kyosai Hospital: Yokohama Sakae Kyosai Byoin

Hideaki Kato

Yokohama Sakae Kyosai Hospital: Yokohama Sakae Kyosai Byoin

Masahiko Kawaguchi

Yokohama Sakae Kyosai Hospital: Yokohama Sakae Kyosai Byoin

\section{Case report}

Keywords: Aberrant right subclavian artery, Esophageal carcinoma, Non-recurrent inferior laryngeal nerve, Semi-prone, Thoracoscopic esophagectomy

Posted Date: September 17th, 2021

DOI: https://doi.org/10.21203/rs.3.rs-835589/v1

License: (c) (i) This work is licensed under a Creative Commons Attribution 4.0 International License.

Read Full License 


\section{Abstract \\ Background}

Aberrant right subclavian artery (ARSA) accompanied by non-recurrent inferior laryngeal nerve (NRILN) is a rare anomaly. In cases of thoracic esophageal carcinoma associated with ARSA and NRILN, surgeons must take extra care not to injury these vessels and nerves. We believe semi-prone thoracoscopic esophagectomy to be a surgical approach that can safely deal with such an anomaly.

\section{Case presentation:}

A 70-year-old man complained of feelings of chest constriction. Endoscopic examination revealed an esophageal tumor and computed tomography showed an ARSA. we performed semi-prone thoracoscopic esophagectomy for case with ARSA and NRILN. We identified these anomalies during esophagectomy, and we could complete surgery without injury these vessels and nerves. The patient had an uneventful recovery and discharged 22 days after surgery.

\section{Conclusions}

Semi-prone thoracoscopic esophagectomy for esophageal carcinoma can be performed safely with a wide operative field, and is an excellent procedure for dissecting esophageal carcinoma in patients with ARSA and NRILN.

\section{Background}

An aberrant right subclavian artery (ARSA) is a rare anomaly, often accompanied by non-recurrent inferior laryngeal nerve (NRILN), which causes a much higher risk of vessel and nerve injury and concomitant severe adverse events during esophagectomy, including bleeding, hoarseness, and pulmonary complications. We consider semi-prone thoracoscopic esophagectomy to be useful in such cases. The semi-prone position leads to fewer pulmonary complications and blood loss, decrease workload, and provide better ergonomic results in comparison with the left lateral decubitus position. Also, if required, conversion to a thoracotomy in the left decubitus position or supine position is easier from the semiprone than from the prone position. Here, we report a semi-prone thoracoscopic esophagectomy performed to treat esophageal carcinoma with ARSA and NRILN.

\section{Case Presentation}

A 70-year-old man complained of feelings of chest constriction. He was diagnosed as having esophageal cancer by means of endoscopy and was then hospitalized for surgery. Endoscopic examination showed type 1 esophageal carcinoma in the mid-thoracic esophagus (Fig. 1). Contrast-enhanced computed 
tomography revealed esophageal carcinoma in the mid-esophagus, an ARSA branching from the descending aorta (Fig. 2), no enlargement of regional lymph nodes, and no distant metastasis. These findings led to the diagnosis of esophageal carcinoma, and with tumor-node-metastasis classification of cT1NOM0, c-Stage I. The patient had no history of smoking and alcohol consumption. He had a past medical history of diabetes mellitus. Semi-prone thoracoscopic esophagectomy was planned. We placed the patient in the left semi-lateral decubitus position with the right side of his body raised at an angle of $30^{\circ}$ from the prone position and with the bed rotated ventrally. We placed ports in the third, fifth, and seventh intercostal spaces on the posterior axillary line (Fig. 3). After transecting the azygous arch and the right bronchial artery, the right subclavian artery (RSA) was found to branch directly from the descending aorta and travel along the left rear side of the esophagus. We taped the right vagus nerve prior to peeling the recurrent nerve from the esophagus (Fig. 4). Although the esophagus was positioned tightly between the trachea and the subclavian artery, we were able to resect the esophagus at a satisfactory location. Thoracoscopic esophagectomy and mediastinal lymph node dissection were then performed.

The patient was placed in the supine position, for commencement of abdominal surgery. The gastric tube conduit was pulled up through the retrosternal space, and end-to-end anastomosis was performed by hand-suturing in the neck field, preserving the NRILN. The operation time was 365 minutes and the intraoperative blood loss $150 \mathrm{ml}$. Pathological examination revealed moderately differentiated squamous cell carcinoma with no lymph node metastasis. The tumor had invaded the submucosa, with no apparent metastasis of dissected lymph nodes ( $\mathrm{pT} 1 \mathrm{bNOM0}$; $\mathrm{p}$-Stage $\otimes)$. The patient was taken to the intensive care unit while intubated and was subsequently extubated on postoperative day 1 . The thoracic drainage tube was removed on postoperative day 7 , and oral intake was begun on postoperative day 8 . The patient's postoperative course was uneventful, and he was discharged on postoperative day 22.

\section{Discussion And Conclusions}

ARSA is a congenital anomaly that develops from changes in anatomical development of the right fourth arch and the left sixth arch. The right fourth arch usually forms the RSA, and the left sixth arch forms an arterial duct. These vessels then descend into the thoracic cavity. When the right fourth arch disappears in early embryonic development, however, the RSA is formed from the right dorsal aorta and seventh intersegmental artery, and the RSA becomes the fourth branch of the aortic arch [1]. In such a case, because the resulting anatomy does not involve the right laryngeal nerve, the nerve branches from the vagal nerve trunk in the neck and becomes the NRILN [2,3]. ARSA affects approximately $0.5-2 \%$ of the general population and is more frequent in women [4]; the frequency of NRILN associated with ARSA is $0.3-1.6 \%$ [3]. NRILN is not recognizable preoperatively. However, because enhanced computed tomography reveals the ARSA, the existence of NRILN is predictable [5].

Video-assisted thoracoscopic surgery (VATS) has been increasing in frequency worldwide because of minimally invasive surgical approaches and attempts to enlarge the visual field in esophageal cancer surgery. Three fields lymphadenectomy is important in radical surgery for esophageal cancer. VATS 
therefore provides a good surgical field of view for lymph-node dissection and enables a precise operation. In particular, VATS in the prone position enlarges the mediastinum by gravity, and leaking fluids and pooling blood do not interfere with the operative view, making esophagectomy safer $[6,7,8]$. Some reports have suggested that VATS is more technically difficult than open thoracotomy [3]. Moreover, if conversion to open surgery is necessary, it is difficult to change the body position when performing VATS on a patient in the prone position. Therefore, we employ the semi-prone position because it is easier to change the body position to left decubitus position or supine position and convert to open surgery than when the procedure is begun with the patient in prone position. Semi-prone position may overcome this problem with retaining the benefits of prone position.

Additionally, compared with the left lateral decubitus position, the semi-prone position provides a wide surgical field in the posterior mediastinum without lung retraction, and damage to the lung is comparably minimized. Because the semi-prone position eliminates the burden of requiring a skilled assistant, surgeons can more safely perform lymphadenectomy in the posterior mediastinum [6]. Some reports have revealed no difference in the blood loss volume, dissection rate, radical resection rate, perioperative complications, and conversion rate, although the operation time is shorter when using the prone position [7].

Kasashima et al. [8] and Kanaji et al. [9] reported that they successfully and safely performed VATS in the prone position for patients with congenital anomalies. However, we believe that the semi-prone position is more beneficial than the conventional prone position because it is easy to change the body position when conversion to open surgery is required in patients with anatomical anomalies, as in our patient. In a search of the PubMed database, we found no previous reports of semi-prone esophagectomy with concomitant ARSA and NRILN. The present report may be the first to describe the accomplishment of semi-prone thoracoscopic esophagectomy for esophageal carcinoma in a patient with ARSA and NRILN.

\section{Abbreviations}

ARSA, aberrant right subclavian artery; NRILN, non-recurrent inferior laryngeal nerve; RSA, right subclavian artery; VATS, video-assisted thoracoscopic surgery

\section{Declarations}

\section{Ethics approval}

All procedures performed in studies involving human participants were in accordance with the ethical standards of the institutional and/or national research committee and with the 1964 Helsinki declaration and its later amendments or comparable ethical standards.

\section{Consent for publication}


Written informed consent was obtained from the patient for publication of this case report and any accompanying images.

\section{Availability of data and materials}

Not applicable.

\section{Competing interests}

The authors declare that they have no competing interests.

\section{Funding}

Not applicable.

\section{Authors' contributions}

KK: Study conception and manuscript drafting and revision. TW: supervision. HK: data acquisition. MK: data acquisition.

\section{Acknowledgements}

The authors thank Angela Morben, DVM, ELS, and Hugh McGonigle, from Edanz (https://www.jp.edanz.com/ac), for editing a draft of the manuscript.

\section{Authors' information (optional)}

Not applicable.

\section{References}

1. Subasinghe D, Sudasinghe $H$, Keppetiyagama CT, et al. Minithoracotomy oesophagectomy for oesophageal carcinoma with aberrant right subclavian artery: a rare case of dysphagia. BMC Gastroenterol. 2014;14:163-7.

2. Tamachi T, Hoshino I, Kono T, et al. A case of esophageal carcinoma with aberrant right subclavian artery. J Jpn Surg Assoc. 2013;74:2755-60.

3. Mizuuchi $Y$, Watanabe $M$, Suehara N, et al. A case of prone thoracoscopic esophagectomy for esophageal carcinoma with aberrant right subclavian artery and nonrecurrent inferior laryngeal nerve. J Jpn Surg Assoc. 2010;71:654-8.

4. Ujiie $\mathrm{N}$, Kikuchi $\mathrm{H}$, Watanabe $\mathrm{T}$, et al. A case of a right subclavian artery-anastomotic fistula associated with the aberrant subclavian artery developed after radical operation for esophageal cancer. J Jpn Surg Assoc. 2014;75:1836-9. 
5. Niu ZX, Zhang H, Chen LQ, et al. Preoperative computed tomography diagnosis of non-recurrent laryngeal nerve in patients with esophageal carcinoma. Thorac Cancer. 2017;8:46-50.

6. Lin J, Kang M, Chen C, et al. Thoracoscopic oesophageal mobilization during thoracolaparoscopy three-stage oesophagectomy: a comparison of lateral decubitus versus semiprone positions. Interact Cardiovasc Thorac Surg. 2013;17:829-34.

7. Seesing MFJ, Goense L, Ruurda JP, et al. Minimally invasive esophagectomy: a propensity scorematched analysis of semiprone versus prone position. Surg Endosc. 2018;32:2758-65.

8. Kasashima H, Kubo N, Ohira M, et al. Successful Resection of Esophageal Carcinoma with Aberrant Right Subclavian Artery Using Video-assisted Thoracoscopic Surgery: Report of Two Cases. Anticancer Res. 2014;34:899-904.

9. Kanaji S, Nakamura T, Otowa Y, et al. Thoracoscopic esophagectomy in the prone position for esophageal cancer with right aortic arch: case report. Anticancer Res. 2013;33:4515-9.

\section{Figures}




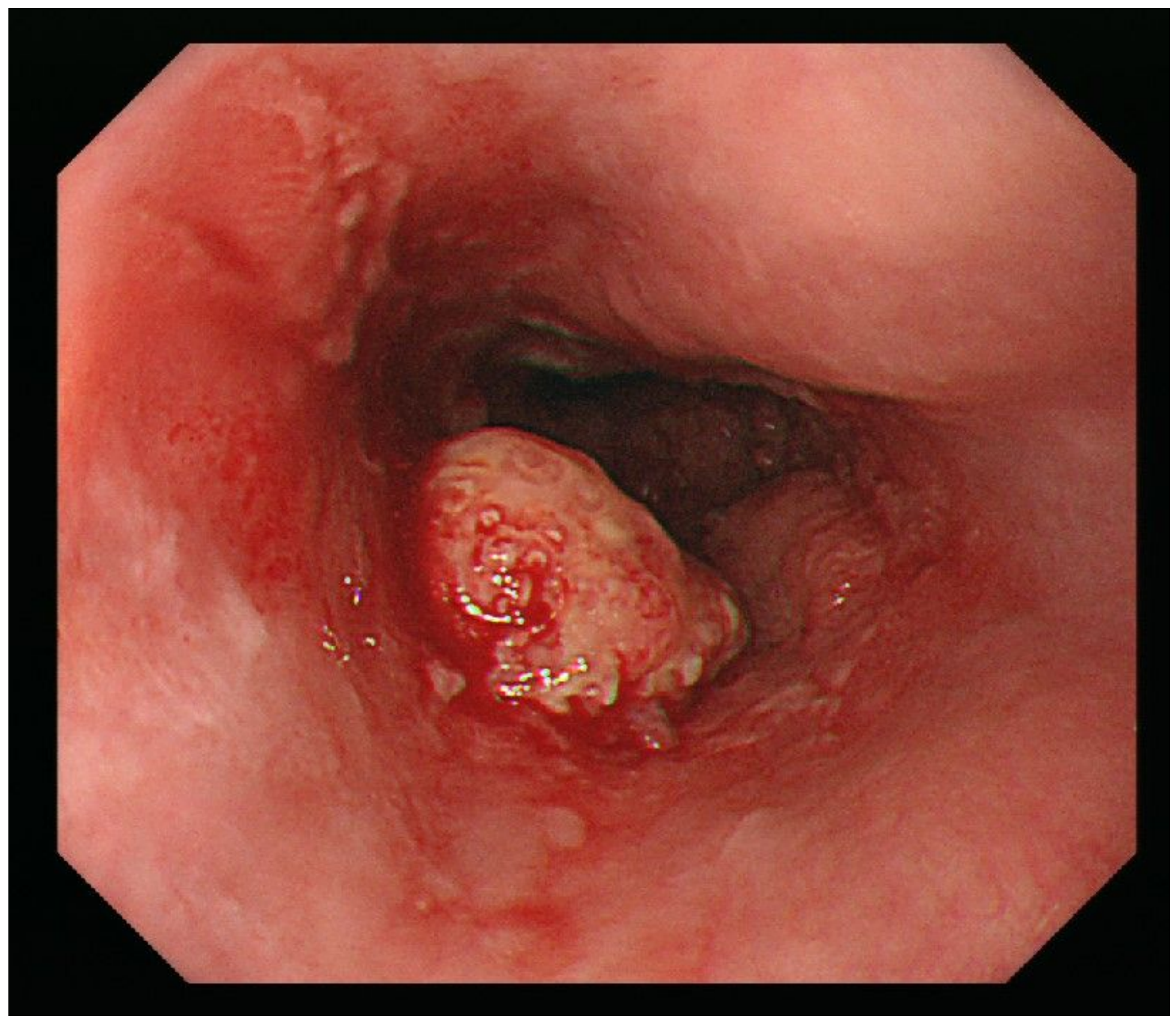

\section{Figure 1}

Photograph taken during endoscopic examination showing type 1 esophageal carcinoma extending from the mid to lower thorax 


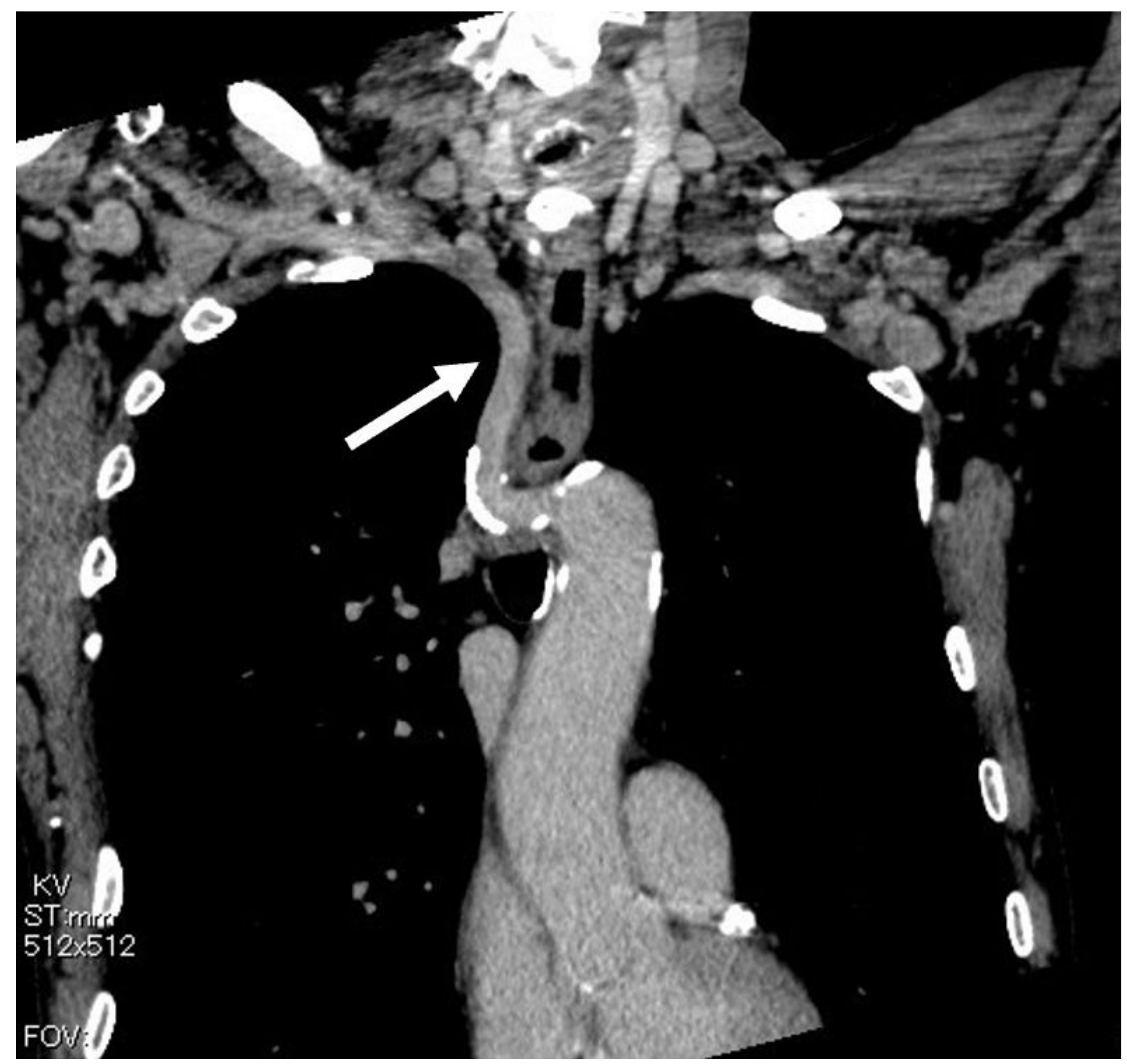

Figure 2

Computed tomographic image showing the aberrant right subclavian artery (arrow) 


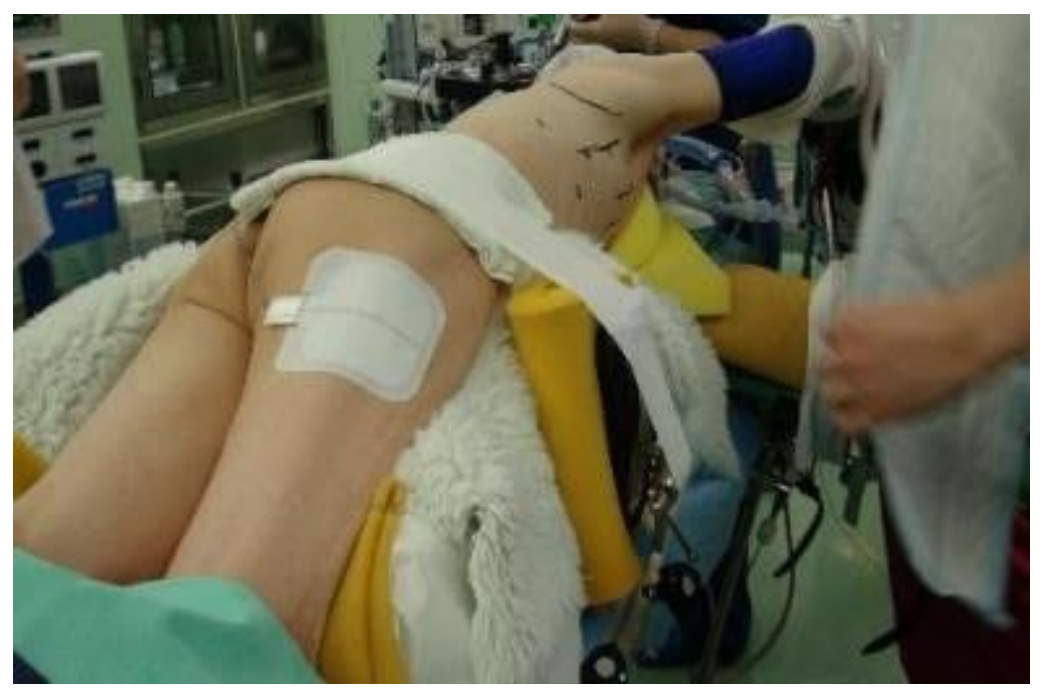

Figure 3

Photograph showing the patient in the semi-prone position

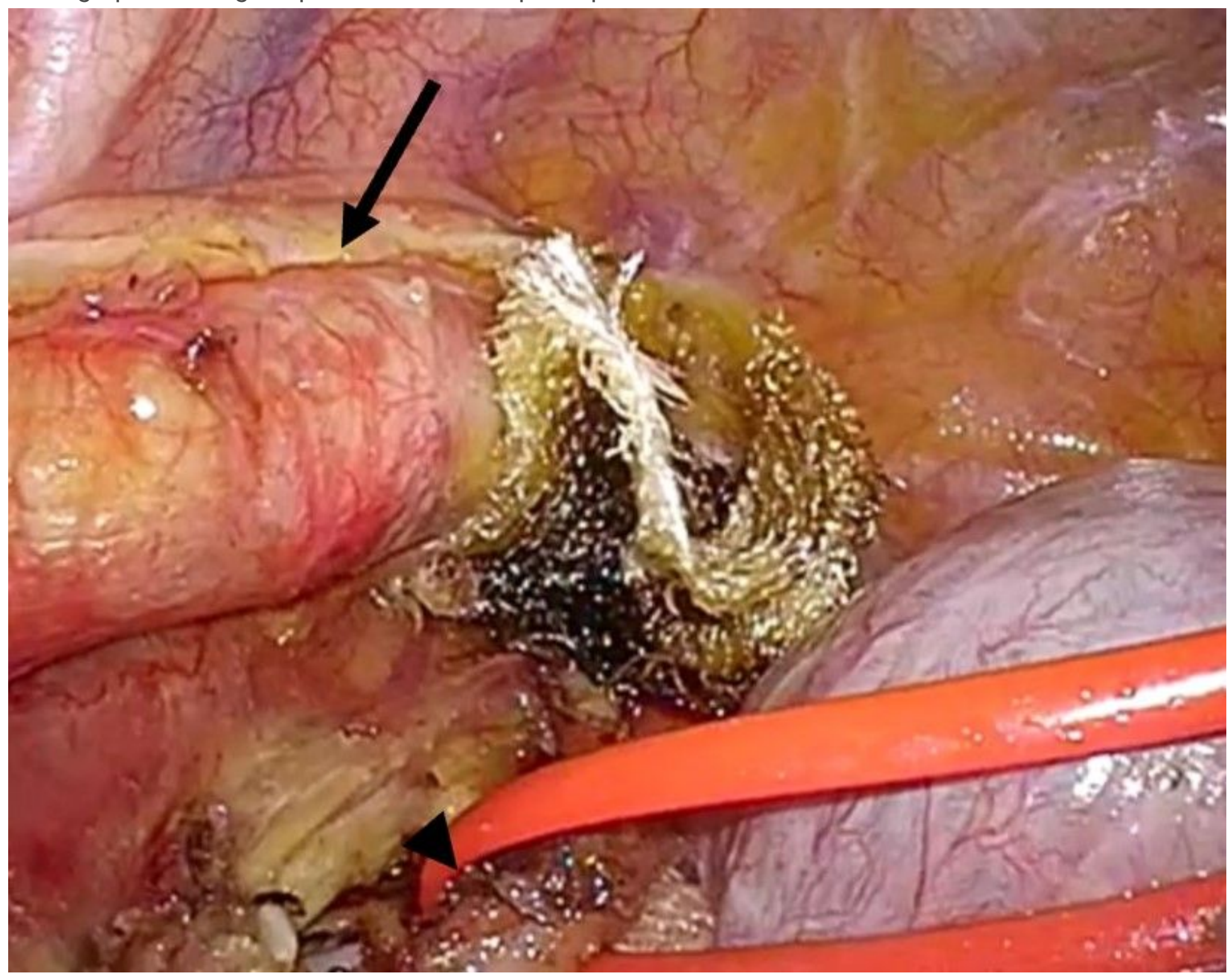


Figure 4

Intraoperative photograph showing the aberrant right subclavian artery (arrow) and the right vagus nerve (arrowhead)

\section{Supplementary Files}

This is a list of supplementary files associated with this preprint. Click to download.

- CAREchecklist.pdf 